\title{
Indistinguishable gene expression between healthy eyes and eyes with unilateral exfoliative glaucoma
}

This article was published in the following Dove Press journal:

Clinical Ophthalmology

\author{
Marcelo Ayala ${ }^{1-3}$ \\ Filip Cuklev ${ }^{4}$ \\ 'Eye Department, Skaraborg Hospital, \\ Skövde, Sweden; ${ }^{2}$ Sahlgrenska \\ Academy, Gothenburg University, \\ Gothenburg, Sweden; ${ }^{3}$ Karolinska \\ Institute, Solna, Sweden; ${ }^{4}$ TATAA \\ Biocenter, Gothenburg, Sweden
}

Background: Glaucoma is an optic neuropathy associated with visual field loss. There are different types of glaucoma, among them exfoliative glaucoma. Glaucoma can present as unilateral or bilateral. The present study aimed to show the association between gene expression and exfoliation in unilateral glaucoma cases.

Methods: Included patients were suffering from exfoliative glaucoma in one eye, meanwhile the other eye was healthy and used as a control. Lens capsule and conjunctival biopsies were taken from both eyes. Gene expression was analyzed.

Results: Both groups were completely different at baseline regarding intraocular pressure, visual acuity before the operation, visual field damage, optic nerve damage, etc. As for gene expression, the only significant difference was found in CYP1B1 from lens capsules. None of the other genes studied showed differential expression in either lens capsules or conjunctival biopsies.

Conclusion: No difference in gene expression was found between eyes with and without exfoliative glaucoma. Exfoliative glaucoma seems to be a bilateral disease, though the phenotype is not always clinically present.

Keywords: exfoliation, glaucoma, gene expression, unilateral

\section{Background}

Glaucoma is a family of eye diseases defined by the progressive loss of retinal ganglion cells. Clinically, glaucoma presents as an optic neuropathy leading to visual field defects. Primary open-angle glaucoma, chronic angle-closure glaucoma, and exfoliative glaucoma (XFG) are the most prevalent forms of glaucoma globally and are the most common causes of glaucoma-related blindness worldwide. It has been described that glaucoma development probably depends on both genetic and environmental factors. ${ }^{1}$

Exfoliation syndrome (XFS) is an accumulation of greyish material in the anterior part of the eye. These deposits are located on the trabecular meshwork and may contribute to elevated intraocular pressure (IOP), optic nerve degeneration, and XFG. The presence of exfoliation material (XFM) seems to be associated not only with glaucoma. It has been increasingly observed in cataract surgery complications and retinal venous occlusive disease. ${ }^{2}$ Deposits of greyish protein material on the anterior lens surface is the most common and essential diagnostic feature of XFS. The classic pattern of exfoliation becomes visible only when the pupil is fully dilated. The exact chemical composition of XFM remains unknown. It has been suggested that overproduction and abnormal metabolism of glycosaminoglycan could induce the presence of XFS. Regardless of etiology, typical XFM has been demonstrated by electron microscopy in different parts of the eye. Exfoliation has been described in the lens epithelium, the non-pigmented
Correspondence: Marcelo Ayala Eye Department, Skaraborg Hospital, 54I 85 Skövde, Sweden

Tel +46500 43l 000

Fax +46500432 I79

Email marcelo.ayala@vgregion.se (c) (1) (8) 2018 Ayala and Cuklev. This work is published and licensed by Dove Medical Press Limited. The full terms of this license are available at https://www.dovepress.com/terms.php cc. hereby accept the Terms. Non-commercial uses of the work are permitted without any further permission from Dove Medical Press Limited, provided the work is properly attributed. For permission for commercial use of this work, please see paragraphs 4.2 and 5 of our Terms (https://www.dovepress.com/terms.php). 
ciliary epithelium, the iris pigment epithelium, the corneal endothelium, the trabecular endothelium, and conjunctiva..$^{3,4}$ XFM deposits at the trabecular meshwork creates increased IOP leading to damage to the optic nerve and visual field defects, ie, XFG. Clinically, it is quite common that patients with XFS reveal only unilateral ocular involvement. However, the generalized nature of the disorder suggests that XFS is probably an asymmetric rather than unilateral condition. ${ }^{5}$ The presence of XFS among patients who were scheduled to undergo cataract operation was unilateral in $41.3 \%$ of the subjects and bilateral in $58.7 \%$, according to a Turkish study. ${ }^{6}$ In a significant cohort study performed in Sweden, $55 \%$ of subjects with unilateral XFS converted to bilateral XFS during a follow-up period of 21 years. $^{7}$

Previous investigations revealed that LOXL1 gene was associated with exfoliation in a population-based study conducted in Iceland. The possible explanation was that products of LOXL1 catalyzed the formation of elastin fibers, found to be a significant component of the lesions in exfoliative glaucoma. ${ }^{8}$ Several other genes have been shown to be associated with the presence of glaucoma, eg, MYOC, OPTN, WDRD36, CYP1B1, GSTM1, and NTF4. ${ }^{1,9-15}$

This study aimed to investigate the association between gene expression of LOXL1, MYOC, OPTN, WDRD36, CYP1B1, GSTM1, and NTF4 genes and the presence of $\mathrm{XFG}$ in unilateral cases. The contralateral healthy eye served as control.

\section{Methods}

Design: nonrandomized cross-sectional control-case study.

Inclusion criteria: patients suffering from XFG in one eye, meanwhile the other eye was healthy (no glaucoma) and free from XFM; both eyes had cataracts which required surgery.

Patients coming to the Eye Department at the Skaraborg Hospital who fulfilled the inclusion criteria were asked to participate in the study. Patients were enrolled between January 2014 and December 2016. Every patient provided written informed consent to participate in the study (including publication of images). The authors have considered the ethical aspects of the research and followed the guidelines of the Helsinki Declaration. The study was approved by the Ethical Committee at the Gothenburg University (approval number: 119-12). Due to ethical reasons, the decision to perform cataract surgery was made before asking patients to participate in the study.

Exclusion criteria: patients with small pupils $(<5 \mathrm{~mm})$; patients with corneal opacities; patients who underwent previous surgeries; patients with poor cooperation under local anesthesia; or patients suffering from conjunctival diseases.

Comprehensive medical and ocular history were obtained for each patient. All patients were examined by the same physician (MA). Included patients were referred to the ophthalmology department due to high IOP detected by an optician. Ophthalmological examinations were performed, and decisions regarding cataract operations were made before including patients in the study. Visual acuity, IOP measurements, gonioscopy, optic nerve status, and the presence or absence of exfoliation were recorded. All patients previously performed visual field tests. Visual acuity was recorded using a Snellen chart. The IOP was measured by Goldmann tonometry three times, and the average was calculated. Gonioscopy was performed in a dark room using a goniolens with undilated pupils, and the anterior chamber angle was classified as 0-4 (according to Shaffer), pigmentation was classified as $0-3$, and the inferior angle was registered. The presence of Sampaolesi's line was also recorded at the inferior angle. The patient's pupils were dilated, and exfoliation was determined to be present or absent. Unilateral exfoliation was defined clinically as the presence of biomicroscopically detectable XFM on the anterior capsule of the lens or at the pupillary border in one eye after pupillary dilatation with $2.5 \%$ phenylephrine and tropicamide $0.5 \%$ (Bausch \& Lomb Incorporated, Bridgewater, NJ, USA). Eyes were classified as "no exfoliation" if there was no evidence of XFM on the pupil, lens or the angle. The optic nerve status was evaluated using a $90 \mathrm{D}$ lens, and the average of vertical cupping was recorded as the cup-to-disc ratio. The optic nerve was also assessed using optical coherence tomography (OCT), (3D OCT-2000; Topcon Corporation, Tokyo, Japan). The protocol used was the $3 \mathrm{D}$ optic disc protocol $(6 \times 6 \mathrm{~mm}$, $512 \times 128)$ which generates images from 128 horizontal linear scans performed by $512 \mathrm{~A}$-scans and measures the retinal nerve fiber layer thickness in a $6 \times 6 \mathrm{~mm}$ area around the optic disc. OCT scans were considered reliable when the reliability index displayed by the machine was $\geq 60$. In case of a lower index, the patient was rechecked, and if the index was still under 60 the patient was excluded from the study. All patients performed repeated visual field testing by Humphrey Field Analyzer (Carl Zeiss Meditec AG, Jena, Germany) using the software threshold 24-2. Only reliable visual fields were considered.

XFG was defined as untreated IOP of $22 \mathrm{mmHg}$ or beyond, open anterior chamber angle in gonioscopy, glaucomatous visual field defect (at least two repeatable Humphrey 24-2 test results), and glaucomatous optic nerve damage, 
concomitant with the presence of XFM, observed at the anterior lens capsule and/or at the pupillary border according to the definition of the European Glaucoma Society. ${ }^{16}$

The healthy contralateral eye was defined as normal IOP ( $\leq 21 \mathrm{mmHg}$ ), normal visual fields (at least two repeatable Humphrey 24-2 test results) and normal optic nerve. Reliable visual fields were defined as $\leq 10 \%$ false positives, $\leq 20 \%$ false negatives, and $\leq 20 \%$ fixation losses. The optic nerve was clinically evaluated using a 90 D lens and OCT. Pupils were dilated, and no exfoliation was present.

The time between XFG diagnosis and the operation were defined as the time between the first occasion XFG was mentioned in the patient's medical records and the date of surgery.

\section{Surgical procedure}

All patients underwent cataract surgery using phacoemulsification technique in both eyes but on different dates. No patient underwent surgery in both eyes on the same day. All operations were performed under local anesthesia by the same surgeon (MA). All surgeries followed our protocol regarding anesthesia and mydriatics. The same protocol was used for patients with and without exfoliation.

A clear corneal incision in the temporal quadrant was made. Then, the anterior chamber was filled with a viscoelastic solution (Duovisc; Alcon Laboratories, Inc., Fort Worth, TX, USA). Continuous curve capsulorrhexis (CCC) was performed using Utrata forceps. CCC was approximately $5-5.5 \mathrm{~mm}$ in diameter. No trypan blue was used to dye the capsule. After CCC, the anterior capsule was put in an Eppendorf tube (Sigma-Aldrich Co., St Louis, MO, USA) containing $0.2 \mathrm{~mL}$ balanced salt solution (BSS) and transported immediately to a freezer $\left(-80^{\circ} \mathrm{C}\right)$ and stored. Then, the rest of the surgery followed the standard steps of phacoemulsification cataract surgery described elsewhere. ${ }^{17}$

After completion of cataract surgery, a conjunctival biopsy was taken from the lower fornix. The biopsy was taken with forceps and Vannas scissors. The biopsy was approximately $16-20 \mathrm{~mm}$ in length and $10 \mathrm{~mm}$ in breadth. The biopsy was put into an Eppendorf tube containing $0.2 \mathrm{~mL} \mathrm{BSS}$ and immediately transported to a freezer $\left(-80^{\circ} \mathrm{C}\right)$ and stored.

\section{RNA extraction, RT and qPCR analysis of human anterior lens capsule and conjunctiva}

Twenty snap-frozen lens capsules and 20 snap-frozen conjunctiva biopsies in individual tubes were shipped on dry ice to TATAA Biocenter (TATAA Biocenter AB, Gothenburg,
Sweden). The samples were stored at $-80^{\circ} \mathrm{C}$ until analysis. Extraction was carried out using RNeasy Micro RNA isolation kit (Qiagen NV, Venlo, the Netherlands) according to the manufacturer's protocol, including carrier and DNAse I treatment. The extracted RNA was reverse transcribed into cDNA using TATAA Grandscript cDNA synthesis kit (TATAA Biocenter AB) according to protocol. Upon qPCR analysis, the cDNA was diluted $10 \times$ for all samples. qPCR was performed using TATAA SYBR Grand Master mix (TATAA Biocenter AB) on Lightcycler480 (Hoffman-La Roche Ltd., Basel, Switzerland) for 12 human reference gene assays from the TATAA Reference Gene Panel (TATAA Biocenter $\mathrm{AB}$ ) and seven validated assays for genes of interest. Also included was the ValidPrime assay (TATAA Biocenter AB) for control of genomic DNA contamination. All information regarding assays and qPCR setup can be found in the Supplementary material according to MIQE guidelines (Tables S1-S4). ${ }^{18}$ The primary analysis of qPCR data was performed with GenEx software (MultiD, Gothenburg, Sweden) using implemented functions for relative quantification. A reference gene screening was performed with Normfinder ${ }^{19}$ to find the optimal reference genes.

\section{Assay validation}

All assays were validated according to the TATAA Biocenter assay validation system. In short, this includes control of specificity on gBlocks ${ }^{\circledR}$ Gene Fragments (Integrated DNA Technologies, Skokie, IL, USA), cDNA, and gDNA. The melt curves were controlled for single peaks, and the generated PCR product was checked for the correct fragment size by capillary electrophoresis. Assay efficiency was determined by a seven-point standard curve down to 20 copies.

\section{RT-input}

The total RNA concentration was below quantification limit for spectrophotometric measurement $(<5 \mathrm{ng} / \mu \mathrm{L})$, thus the reverse transcription input was not known but did not exceed input limitations. The resulting cDNA yield was expected to be sufficient for the current qPCR experiments, and accordingly, the $\mathrm{Cq}$-values were in an acceptable range $(\mathrm{Cq}<30)$ in all samples for most screened reference genes (Tables S1-S4).

\section{Data analysis}

Technical replicates were averaged. The data were corrected for genomic background with ValidPrime (did not change the Cq-values significantly). The data were normalized to reference genes (GAPDH and $\mathrm{UBC}$ ). Each right eye was 
normalized to its corresponding left eye. Relative expression to the maximum was set (lowest expression was set to 1). Log2 was applied to data.

\section{Statistics}

Statistical analysis was performed using the SPSS software (IBM Corporation, Armonk, NY, USA). For the baseline analysis, first, a Kolmogorov-Smirnov analysis was performed, in case of normality, Student's $t$-test was used. Analysis of gene expression was done using the paired samples Student's $t$-test method in case the KolmogorovSmirnov test showed normality, otherwise a non-parametric Mann-Whitney test was used.

\section{Results}

All included patients were born in Sweden, and all patients' parents were born in Sweden. The average age of the included patients at the time of surgery was $78.8 \pm 4.9$ years. Gender distribution was six women and four men. In all cases, the eye showing glaucoma was operated on first due to lower visual acuity than the healthy eye. The mean time between cataract operations (always XFG first) was $3.7 \pm 1.1$ months. All patients in the glaucoma group were treated with anti-glaucomatous eye drops, the number of medicines was $2.4 \pm 0.5$. No medications were used in the healthy control group.

A photograph of one of the patients included in the study suffering from XFG is shown in Figure 1. The photograph shows the typical patterns presented in XFG. XFM is most prominent at the pupillary border.

The time between glaucoma diagnosis and cataract operation was very variable among the patients included, the average time was $4.5 \pm 2$ years.

Table 1 shows comparisons between the groups at baseline. All comparisons at baseline were significant. The mean visual

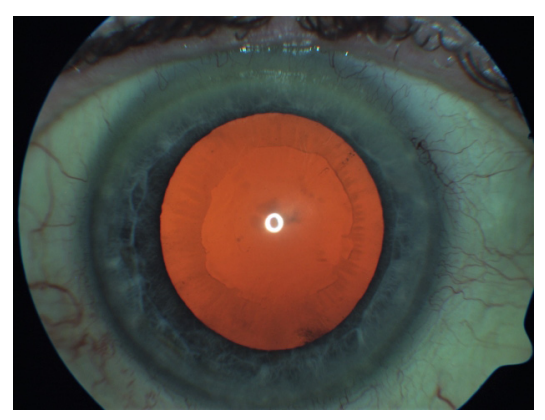

Figure I Photograph showing the right eye of a patient included in the study suffering from exfoliative glaucoma.

Notes: Exfoliation material is seen as a margin on the lens surface. The pupil has been dilated. acuity before the operation was lower in the eyes suffering from exfoliation $(0.34)$ than in the contralateral eyes with cataracts only $(0.52)(p=0.001)$. It is possible that a reduction in visual acuity in glaucoma patients was not only due to cataracts. The mean IOP was normal in the contralateral healthy eyes $(16.3 \mathrm{mmHg})$, meanwhile it was border-line $(21.2 \mathrm{mmHg})$ in the eyes with glaucoma. All glaucoma patients were receiving IOP-reducing treatment. The visual fields in the glaucoma patients showed a mean deviation (MD) of $-9.9 \mathrm{~dB}$ and visual field index of $70.2 \%$, revealing that the included patients suffered from moderate glaucoma. The gonioscopy showed open angle in both groups but a significantly increased pigmentation in the XFG patients $(p<0.001)$.

Regarding gene expression in the lens capsule and conjunctival biopsies, the only gene that showed a significant result was CYP1B1 in the lens capsule. The rest of the genes in both lens capsule and conjunctival biopsies showed no significant comparisons (Tables 2 and 3).

\section{Discussion}

The present study showed no difference in gene expression of several genes between eyes suffering from XFG versus contralateral healthy eyes of the same patients.

XFS is an accumulation of greyish material in the anterior part of the eye. These deposits may contribute to elevated IOP, optic nerve degeneration, and glaucoma, ie, XFG. Deposits of greyish protein material on the anterior lens surface is the most common and essential diagnostic feature of XFS. XFG has been defined as open-angle glaucoma usually presenting with high IOP levels. The nuclear gene associated with the presence of exfoliation is the LOXL1. Thorleifsson et al first described the importance of this gene in $2007 .{ }^{8}$ The authors described two single-nucleotide polymorphisms (SNPs) of the gene LOXL1 that could explain the association with exfoliation. The data suggest that LOXL1 could confer risk of XFG through XFS. The product of LOXL1 catalyzes the formation of elastin fibers, found to be a significant component of the lesions in XFG. Thorleifsson et al studied an Icelandic population. Several reports followed showing different levels of association between the presence of exfoliation and LOXL1 related to ethnicity. A meta-analysis performed by Wang et $\mathrm{al}^{20}$ showed different results comparing studies conducted in several countries. SNP rs1048661 was found to be associated with $\mathrm{XFS} / \mathrm{XFG}$, but the risk allele in white populations was found to be $G$, as opposed to the T allele in Japanese, Chinese, and Korean populations. The SNP rs3825942 was significantly associated with XFS in a black South African 
Table I Baseline characteristics of the included patients

\begin{tabular}{|c|c|c|c|}
\hline Variables & $\begin{array}{l}\text { Eyes with exfoliative } \\
\text { glaucoma } \\
(n=10)\end{array}$ & $\begin{array}{l}\text { Eyes without exfoliative } \\
\text { glaucoma } \\
(n=10)\end{array}$ & $\begin{array}{l}\text { p-value } \\
\text { (Student's t-test) }\end{array}$ \\
\hline Mean visual acuity before operation & 0.34 & 0.52 & 0.001 \\
\hline Mean IOP (mmHg) & 21.2 & 16.3 & 0.0001 \\
\hline Visual fields MD (dB) & -9.9 & -0.58 & $<0.0001$ \\
\hline Visual fields VFI (\%) & 70.4 & 98.5 & $<0.0001$ \\
\hline Mean RNFL thickness OCT $(\mu \mathrm{m})$ & 72.7 & 99.4 & $<0.0001$ \\
\hline Cup-to-disc ratio (0-0.99) & 0.78 & 0.55 & $<0.0001$ \\
\hline Gonioscopy; anterior chamber angle $(0-4)$ & 2.7 & 1.8 & 0.0003 \\
\hline Gonioscopy: pigmentation (0-3) & 2.4 & 0.7 & $<0.0001$ \\
\hline
\end{tabular}

Abbreviations: IOP, intraocular pressure; MD, mean deviation; VFI, visual field index; RNFL, retinal nerve fiber layer; OCT, optical coherence tomography.

population. Several studies have been published showing a correlation between ethnicity and prevalence of LOXL1 in association with exfoliation. ${ }^{21-23}$ No study was found in the literature that included patients coming from Sweden, but it is possible to speculate that ethnicity in Sweden resembles the Icelandic ethnicity. All patients included in the present study were born in Sweden, and their parents were also born in Sweden. However, in the present study, LOXL1 expression at mRNA level was similar when comparing affected and unaffected eyes. These results correlate well with results published by Schöltzer-Schehardt et $\mathrm{al}^{24}$ that showed no difference in mRNA expression comparing enucleated eyes with versus without exfoliation at more advanced stages. Included patients in the present study were all patients suffering from XFG and not only showing exfoliation, thus, no patient at an early stage of exfoliation was included. Other factors beyond gene expression can probably explain the presence or absence of XFM. Local environmental factors like chemical compounds at the anterior chamber of the eye can probably influence the development of XFM.

A significant issue to discuss is the fact of unilateral versus bilateral exfoliation. Is XFM one-sided? Is it possible

Table 2 Gene expression in conjunctival biopsies comparing healthy individuals versus exfoliative glaucoma patients

\begin{tabular}{lllll}
\hline Gene & Healthy & $\begin{array}{l}\text { Exfoliative } \\
\text { glaucoma }\end{array}$ & Test & P-value \\
& & I & \\
\hline LOXL-I $(\bar{x} \pm \mathrm{SD})$ & $10.22 \pm 0.91$ & $10.01 \pm 0.79$ & Student's $t$-test & $0.6 \mathrm{I}$ \\
NTF4 $(\bar{x} \pm \mathrm{SD})$ & $9.27 \pm 0.83$ & $9.30 \pm 0.92$ & Student's $t$-test & 0.94 \\
OPT-N $(\bar{x} \pm \mathrm{SD})$ & $3.47 \pm 0.25$ & $3.52 \pm 0.30$ & Student's t-test & 0.73 \\
WDR36 $(\bar{x} \pm \mathrm{SD})$ & $5.14 \pm 0.35$ & $5.17 \pm 0.29$ & Student's t-test & 0.86 \\
CYPIBI $(\bar{x} \pm \mathrm{SD})$ & $6.98 \pm 0.95$ & $6.30 \pm 1.30$ & Student's t-test & 0.23 \\
\hline
\end{tabular}

Notes: Data analysis: I) genes with more than $50 \%$ missing data were removed (hMYOC and hGSTMI); 2) technical replicates were averaged; 3) the data were corrected for genomic background with ValidPrime (did not change the Cq-values significantly, except for some samples in hNTF4); 5) the data were normalized to reference genes (PPIA and UBC); 6) each diseased eye was normalized to its corresponding healthy eye; 7) Log2 was applied to data. that we cannot detect exfoliation clinically even though XFM is present? These questions have been raised in previous studies. Hammer et $\mathrm{al}^{5}$ described ultrastructural changes in lenses that were clinically unaffected by exfoliation even when the fellow eye was affected. Ultrastructural alterations were observed in anterior segment tissues of all apparently not involved fellow eyes. These included deposits of exfoliation on the iris and ciliary epithelia and in the dilator muscle of the iris; increased accumulation of extracellular matrix and degenerative changes of the iris pigment epithelium. Similar findings were described by Parekh et al, ${ }^{4}$ they studied 32 patients showing clinically unilateral exfoliation and found ultrastructural changes in the conjunctiva and lens capsules in approximately $80 \%$ of eyes. The authors concluded that XFS must be described as a bilateral disorder with a clinically marked asymmetric manifestation. The present study seems to confirm previous ultrastructural results, but at gene expression level.

XFS is an age-related disorder with an estimated prevalence ranging from $10 \%$ to $20 \%$ of the general population

Table 3 Gene expression in the lens capsule comparing healthy individuals versus glaucoma patients

\begin{tabular}{lllll}
\hline Gene & Healthy & $\begin{array}{l}\text { Exfoliative } \\
\text { glaucoma }\end{array}$ & Test & p-value \\
\hline LOXL-I $(\bar{x} \pm S D)$ & $7.0 I \pm 0.89$ & $6.78 \pm 0.38$ & Student's $t$-test & 0.42 \\
NTF4 $(\bar{x} \pm S D)$ & $I I .94 \pm 0.78$ & $12.67 \pm 1.05$ & Student's $t$-test & 0.15 \\
OPT-N $(\bar{x} \pm S D)$ & $2.97 \pm 0.26$ & $2.80 \pm 0.37$ & Student's $t$-test & 0.22 \\
WDR36 ( $\bar{x} \pm S D)$ & $5.58 \pm 0.15$ & $5.38 \pm 0.43$ & Student's $t$-test & 0.16 \\
CYPIBI $(\bar{x} \pm S D)$ & $4.79 \pm 0.90$ & $3.99 \pm 0.97$ & Student's $t$-test & $0.04 *$ \\
MYOC $(\bar{x} \pm S D)$ & $5.94 \pm 1.10$ & $6.54 \pm 0.91$ & Student's $t$-test & 0.16 \\
GSTMI $(\bar{x} \pm S D)$ & $7.38 \pm 4.94$ & $7.84 \pm 5.02$ & Mann-Whitney & 0.52 \\
\hline
\end{tabular}

Notes: Data analysis: I) technical replicates were averaged; 2) the data were corrected for genomic background with ValidPrime (did not change the Cq-values significantly); 3) the data were normalized to reference genes (GAPDH and UBC); 4) each right eye was normalized to its corresponding left eye; 5) relative expression to the maximum was set (lowest expression was set to I); 6) Log2 was applied to data. *Comparison was significant. 
over 60 years of age. ${ }^{3}$ The presence of the condition varies among different countries. Tarkkanen and Kivela ${ }^{25}$ published a retrospective study in 2004 including 35 patients with clinically unilateral XFS. The authors showed that the median age at conversion to bilateral XFS was 72 years (range, 66-86). However, $48 \%$ of patients remained unilaterally affected in a 15-year follow-up period. In the Reykjavik Eye Study, it was reported that $71 \%$ of patients converted to bilateral in 12-year follow-up period. ${ }^{26}$ Aström et al $^{7}$ reported a conversion rate of $55 \%$ in northern Sweden in a 21 -year follow-up period. Puska ${ }^{27}$ described a conversion rate of $38 \%$ from no exfoliation to exfoliation and a $32 \%$ rate considering XFG in 10 -year follow-up. The patients included in our study were operated on when they were aged approximately 78.8 years on average, meanwhile the diagnosis of XFG was reported 4.5 years before surgery. Our patients were in general older than patients included in previous studies. The reason could be that we included patients suffering from XFG and not only exfoliation. It takes several years to develop XFG with a probably significant individual variation. Furthermore, all previously described studies included patients showing exfoliation only and not suffering from XFG. Unfortunately, no evidence in the literature was found about time conversion from unilateral to bilateral XFG.

In our study, the only gene that showed a slight difference in gene expression between XFG and healthy eyes was CYP1B1 isolated in the lens capsule. This gene encodes a member of the cytochrome P450 superfamily of enzymes. The cytochrome $\mathrm{P} 450$ proteins are monooxygenases which catalyze many reactions involved in drug metabolism, synthesis of cholesterol and other lipids. Mutations in CYP1B1 have been associated with primary congenital glaucoma; therefore it is thought that the enzyme also metabolizes a signaling molecule involved in eye development. ${ }^{28,29}$ Results coming from our study must be interpreted with caution, the level of significance was not high $(p=0.04)$ and the sample size was not big. The role of CYP1B1 in exfoliation is not clear; previous studies postulated that this gene could be related to oxidation processes. ${ }^{30,31}$ Although the specific pathogenesis of exfoliation remains unknown, data support a pathogenic concept of exfoliation being a type of stress-induced elastosis. This process has been associated with the excessive production of an abnormal cross-linking of elastic fibers. ${ }^{32}$ Increased oxidative stress has been identified as a potential cause of exfoliation; ${ }^{33}$ it is possible to speculate that CYP1B1 can mediate exfoliation through oxidative stress. Further investigations are needed to clarify the role of CYP1B1 in exfoliation.
A strength of the present study is that all patients were diagnosed with XFG several years before inclusion, the diagnosis was based both on structural and functional tests. Functional tests like repeated Humphrey visual fields and structural tests like the clinical observation of the optic nerve and measurements with OCT device were used. Furthermore, all surgeries both in exfoliation and no exfoliation patients were performed by the same surgeon (MA), diminishing the risk of bias induced by several surgeons.

The present study has several limitations. The sample size was not so big. However, the study design was quite unusual, we used the contralateral eye as control, in this way we were quite sure that the reason for no clinical presentation of exfoliation could not be attributed to the DNA level, both eyes have the same DNA. The reason for no clinical appearance of exfoliation might be due to mRNA or protein level. The significant majority of the previously published studies related to exfoliation and genes were based on blood samples. Studies considered gene expression at the DNA level and compared affected versus unaffected individuals. No similar studies testing gene expression at the mRNA level comparing the same individuals were found in the literature. To further support the results of the current study, in addition to increasing the physiological understanding, changes in protein level should be studied.

Another limitation of the present study is that cases with very advanced exfoliation and small pupils were not included. The reason for exclusion is that in these cases the surgical procedure is different, it is necessary to use iris hooks and trypan blue that could alter the results of gene expression measurements. However, the included patients had moderate glaucoma $(\mathrm{MD}=9.9 \mathrm{~dB})$, the XFM might have been sufficient to perform analysis in the affected eyes.

In summary, the present study supports results from previous ultrastructural studies showing that exfoliation is an asymmetrical clinically expressed disorder instead of a single condition. Local factors at the anterior chamber of the eye might either expedite exfoliation or slow it down in one eye, explaining the asymmetric clinical presentation.

\section{Acknowledgment}

The authors thank the Västra Götaland Region and the Swedish Glaucoma Association for financial support.

\section{Disclosure}

The authors report no conflicts of interest in this work.

\section{References}

1. Liu Y, Allingham RR. Molecular genetics in glaucoma. Exp Eye Res. 2011;93(4):331-339. 
2. Pasquale LR, Borras T, Fingert JH, Wiggs JL, Ritch R. Exfoliation syndrome: assembling the puzzle pieces. Acta Ophthalmol. 2016;94(6): e505-e512.

3. Ritch R, Schlotzer-Schrehardt U. Exfoliation syndrome. Surv Ophthalmol. 2001;45(4):265-315.

4. Parekh P, Green WR, Stark WJ, Akpek EK. Electron microscopic investigation of the lens capsule and conjunctival tissues in individuals with clinically unilateral pseudoexfoliation syndrome. Ophthalmology. 2008;115(4):614-619.e612.

5. Hammer T, Schlotzer-Schrehardt U, Naumann GO. Unilateral or asymmetric pseudoexfoliation syndrome? An ultrastructural study. Arch Ophthalmol. 2001;119(7):1023-1031.

6. Sekeroglu MA, Bozkurt B, Irkec M, et al. Systemic associations and prevalence of exfoliation syndrome in patients scheduled for cataract surgery. Eur J Ophthalmol. 2008;18(4):551-555.

7. Aström S, Stenlund H, Lindén C. Incidence and prevalence of pseudoexfoliations and open-angle glaucoma in northern Sweden: II. Results after 21 years of follow-up. Acta Ophthalmol Scand. 2007;85(8): $832-837$.

8. Thorleifsson G, Magnusson KP, Sulem P, et al. Common sequence variants in the LOXL1 gene confer susceptibility to exfoliation glaucoma. Science. 2007;317(5843):1397-1400.

9. Rezaie T, Child A, Hitchings R, et al. Adult-onset primary open-angle glaucoma caused by mutations in optineurin. Science. 2002;295(5557) 1077-1079.

10. Chi ZL, Yasumoto F, Sergeev Y, et al. Mutant WDR36 directly affects axon growth of retinal ganglion cells leading to progressive retinal degeneration in mice. Hum Mol Genet. 2010;19(19):3806-3815.

11. Pasutto F, Matsumoto T, Mardin CY, et al. Heterozygous NTF4 mutations impairing neurotrophin-4 signaling in patients with primary open-angle glaucoma. Am J Hum Genet. 2009;85(4):447-456.

12. Guo H, Li M, Wang Z, Liu Q, Wu X. Association of MYOC and APOE promoter polymorphisms and primary open-angle glaucoma: a metaanalysis. Int J Clin Exp Med. 2015;8(2):2052-2064

13. Johnson EC, Guo Y, Cepurna WO, Morrison JC. Neurotrophin roles in retinal ganglion cell survival: lessons from rat glaucoma models. Exp Eye Res. 2009;88(4):808-815.

14. Malik MA, Gupta V, Shukla S, Kaur J. Glutathione S-transferase (GSTM1, GSTT1) polymorphisms and JOAG susceptibility: a case control study and meta-analysis in glaucoma. Gene. 2017;628:246-252.

15. Kumar A, Basavaraj MG, Gupta SK, et al. Role of CYP1B1, MYOC, OPTN, and OPTC genes in adult-onset primary open-angle glaucoma: predominance of CYP1B1 mutations in Indian patients. Mol Vis. 2007; 13:667-676.

16. European Glaucoma Society. Treatment principles and options. In: Terminology and guidelines for glaucoma. 3rd ed. Savona, Italy: Dogma; 2008:117-169.

17. Fine IH, Maloney WF, Dillman DM. Crack and flip phacoemulsification technique. J Cataract Refract Surg. 1993;19(6):797-802.

18. Bustin SA, Benes V, Garson JA, et al. The MIQE guidelines: minimum information for publication of quantitative real-time PCR experiments. Clin Chem. 2009;55(4):611-622.
19. Andersen CL, Jensen JL, Orntoft TF. Normalization of real-time quantitative reverse transcription-PCR data: a model-based variance estimation approach to identify genes suited for normalization, applied to bladder and colon cancer data sets. Cancer Res. 2004;64(15):5245-5250.

20. Wang L, Yu Y, Fu S, Zhao W, Liu P. LOXL1 gene polymorphism with exfoliation syndrome/exfoliation glaucoma: a meta-analysis. J Glaucoma. 2016;25(1):62-94.

21. Anastasopoulos E, Coleman AL, Wilson MR, et al. Association of LOXL1 polymorphisms with pseudoexfoliation, glaucoma, intraocular pressure, and systemic diseases in a Greek population. The Thessaloniki eye study. Invest Ophthalmol Vis Sci. 2014;55(7):4238-4243.

22. Founti P, Haidich AB, Chatzikyriakidou A, et al. Ethnicity-based differences in the association of LOXL1 polymorphisms with pseudoexfoliation/pseudoexfoliative glaucoma: a meta-analysis. Ann Hum Genet. 2015;79(6):431-450.

23. Yilmaz SG, Palamar M, Onay H, et al. LOXL1 gene analysis in Turkish patients with exfoliation glaucoma. Int Ophthalmol. 2016;36(5): 629-635.

24. Schlotzer-Schrehardt U, Pasutto F, Sommer P, et al. Genotype-correlated expression of lysyl oxidase-like 1 in ocular tissues of patients with pseudoexfoliation syndrome/glaucoma and normal patients. Am J Pathol. 2008;173(6):1724-1735.

25. Tarkkanen A, Kivela T. Cumulative incidence of converting from clinically unilateral to bilateral exfoliation syndrome. J Glaucoma. 2004;13(3):181-184.

26. Arnarsson A, Sasaki H, Jonasson F. Twelve-year incidence of exfoliation syndrome in the Reykjavik Eye Study. Acta Ophthalmol. 2013;91(2): 157-162.

27. Puska PM. Unilateral exfoliation syndrome: conversion to bilateral exfoliation and to glaucoma: a prospective 10-year follow-up study. J Glaucoma. 2002;11(6):517-524.

28. Gronskov K, Redo-Riveiro A, Sandfeld L, et al. CYP1B1 Mutations in individuals with primary congenital glaucoma and residing in Denmark. J Glaucoma. 2016;25(12):926-930.

29. Garcia-Anton MT, Salazar JJ, de Hoz R, et al. Goniodysgenesis variability and activity of CYP1B1 genotypes in primary congenital glaucoma. PLoS One. 2017;12(4):e0176386.

30. McLellan RA, Oscarson M, Hidestrand M, et al. Characterization and functional analysis of two common human cytochrome P450 1B1 variants. Arch Biochem Biophys. 2000;378(1):175-181.

31. Stoilov I, Akarsu AN, Sarfarazi M. Identification of three different truncating mutations in cytochrome P4501B1 (CYP1B1) as the principal cause of primary congenital glaucoma (Buphthalmos) in families linked to the GLC3A locus on chromosome 2p21. Hum Mol Genet. 1997;6(4):641-647.

32. Schlotzer-Schrehardt U, Naumann GO. Ocular and systemic pseudoexfoliation syndrome. Am J Ophthalmol. 2006;141(5):921-937.

33. Yagci R, Gurel A, Ersoz I, et al. Oxidative stress and protein oxidation in pseudoexfoliation syndrome. Curr Eye Res. 2006;31(12):1029-1032. 


\section{Supplementary materials}

Table SI Genes of interest

\begin{tabular}{lll}
\hline Gene & FWD-primer & REV-primer \\
\hline LOXLI & GTGGGGAGCGGAACGG & TGCTTGCACATAGTTGGGGT \\
MYOC & GGAGGTAGCAAGGCTGAGAA & CTGGAAGGCCAAAGTGTCCAA \\
OPTN & CATGGGGCGAGAACAAGTGA & GGGGCAGGAATGAATCGGAA \\
WDRD36 & GGCTTTTAGTCCTGATGGTCGT & GAGGAGCCGAGTCCAACAAA \\
CYPIBI & AACGTACCGGCCACTATCAC & TGATCCAATTCTGCCTGCACT \\
GSTMI & CCTCCACCGTATATTTGAGCC & TGAGAACACAGGTCTTGGGA \\
NTF4 & CTGCAAGGCTGATAACGCTG & TCAGATACCCAGTGCCTCCT \\
\hline
\end{tabular}

Abbreviations: FWD, forward; REV, reverse.

Table S2 TATAA human reference gene panel and ValidPrime

\begin{tabular}{lll}
\hline Gene & FWD-primer & REV-primer \\
\hline ACTB & AGAAAATCTGGCACCACACC & AGAGGCGTACAGGGATAGCA \\
B2M & GGGTTTCATCCATCCGACA & ACACGGCAGGCATACTCATC \\
GAPDH & CAGTCAGCCGCATCTTCTTTT & TGGCAACAATATCCACTTTACCAG \\
GUSB & GATGGAAGAAGTGTGCGTAG & TTGCTCACAAAGGCACAGG \\
HPRT & TGACACTGGCAAAACAATGCA \\
UBC & CGCAGTTCTTGTTTGTGGA & GGTCCTTTTCACCAGCAAGCT \\
PPIA & AGGTCCCAAAGACAGCAGAA & GGGTGGACTCTTTCTGGATG \\
RPLP & TCAACATCTCCCCCTTCTCC & CACCACCCTGACACATAAACC \\
I8S & GACTCAACACGGGAAACCTC \\
TBP & TTCGGAGAGTTCTGGGATTG & GCAGACACACTGGCAACA \\
TUBB5 & ATTCAGACACCACTCCACCAAC \\
YWHAZ & AGACGGAAGGTGCTGAGAAA & ATCAGTGCCGTGGTTCGT \\
ValidPrime & AGCACATTTCTATTCTCCGT & ACTCCTTCCTCACCACATCC \\
\hline
\end{tabular}

Abbreviations: FWD, forward; REV, reverse.

Table S3 Quantitative PCR setup

\begin{tabular}{llll}
\hline Program & Temperature $\left({ }^{\circ} \mathbf{C}\right)$ & Time $(s)$ & Cycles \\
\hline Polymerase activation & 95 & 60 & $\mathrm{I}$ \\
Denaturation & 95 & 5 & \\
Annealing & 60 & 30 & 45 \\
Extension & 72 & 10 & \\
Melt curve & $60-95$ & - & - \\
\hline
\end{tabular}

Table S4 Quantitative PCR reaction mix

\begin{tabular}{ll}
\hline Reagent & Volume $(\mu \mathrm{L})$ \\
\hline Primers (Fwd + Rev) & 0.4 \\
TATAA SYBR Grandmaster mix & 5 \\
$\mathrm{H}_{2} \mathrm{O}$ & 2.5 \\
cDNA & 2 \\
Total & 10 \\
\hline
\end{tabular}

Clinical Ophthalmology

\section{Publish your work in this journal}

Clinical Ophthalmology is an international, peer-reviewed journal covering all subspecialties within ophthalmology. Key topics include: Optometry; Visual science; Pharmacology and drug therapy in eye diseases; Basic Sciences; Primary and Secondary eye care; Patient Safety and Quality of Care Improvements. This journal is indexed on

Submit your manuscript here: http://www.dovepress.com/clinical-ophthalmology-journal

\section{Dovepress}

PubMed Central and CAS, and is the official journal of The Society of Clinical Ophthalmology (SCO). The manuscript management system is completely online and includes a very quick and fair peer-review system, which is all easy to use. Visit http://www.dovepress.com/ testimonials.php to read real quotes from published authors. 